\title{
A wideband plasmonic CPW fed optical antenna
}

\author{
N. P. Pathak*, Rohitashv Bansal, Amarjit Kumar and N. P. Pathak \\ RFIC Research Group, Electronics and Communication Engineering Department, \\ Indian Institute of Technology Roorkee, Roorkee, Uttrakhand, India \\ *Email: nagppfec@iitr.ac.in
}

In this paper we report a comparative study of the characteristic impedance for the nanostrip waveguide (NSW) [1] and the plasmonic coplanar waveguide (CPW). The coplanar structures have been received as a promising candidate for an alternative to the nanostrip technology [2] and have a wide range of realizable impedance in comparison to NSW. Impedance range is $(8 \Omega-30 \Omega)$ in the case of

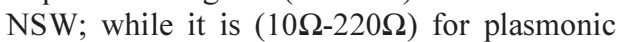
CPW. Plasmonic waveguides supporting subwavelength transmission and manipulation are the essential structures to enable incorporation of ultra-compact plasmonic devices.

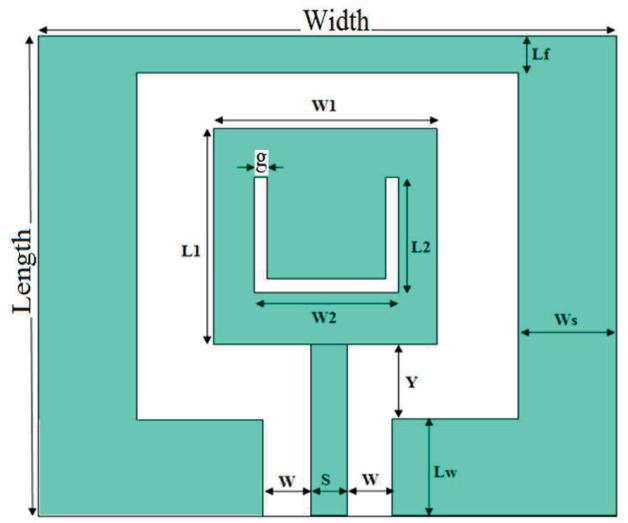

Figure 1: Structure of wideband Plasmonic CPW fed optical antenna

A wide band Plasmonic CPW fed rectangular patch antenna for optical communication is also presented in this paper. The antenna has compact size of $1000 \mathrm{~nm} \times 1000 \mathrm{~nm}$. The proposed optical antenna emits a directional beam with good directivity and radiation efficiency. The proposed antenna has a very wide bandwidth which is useful to communicate in all optical windows. Proposed wideband plasmonic $\mathrm{CPW}$ fed optical antenna structure is shown in Figure 1. The simulated characteristics of the proposed wideband plasmonic CPW fed optical antenna are shown in Figure 2. The simulation results show that the radiation efficiency of proposed antenna is $82 \%$ at $1550 \mathrm{~nm}$ and $51.32 \%$ at $1350 \mathrm{~nm}$ wavelength. The directivity at $1550 \mathrm{~nm}$ is 4.03 $\mathrm{dBi}$ and at $1350 \mathrm{~nm}$ is $3.74 \mathrm{dBi}$.
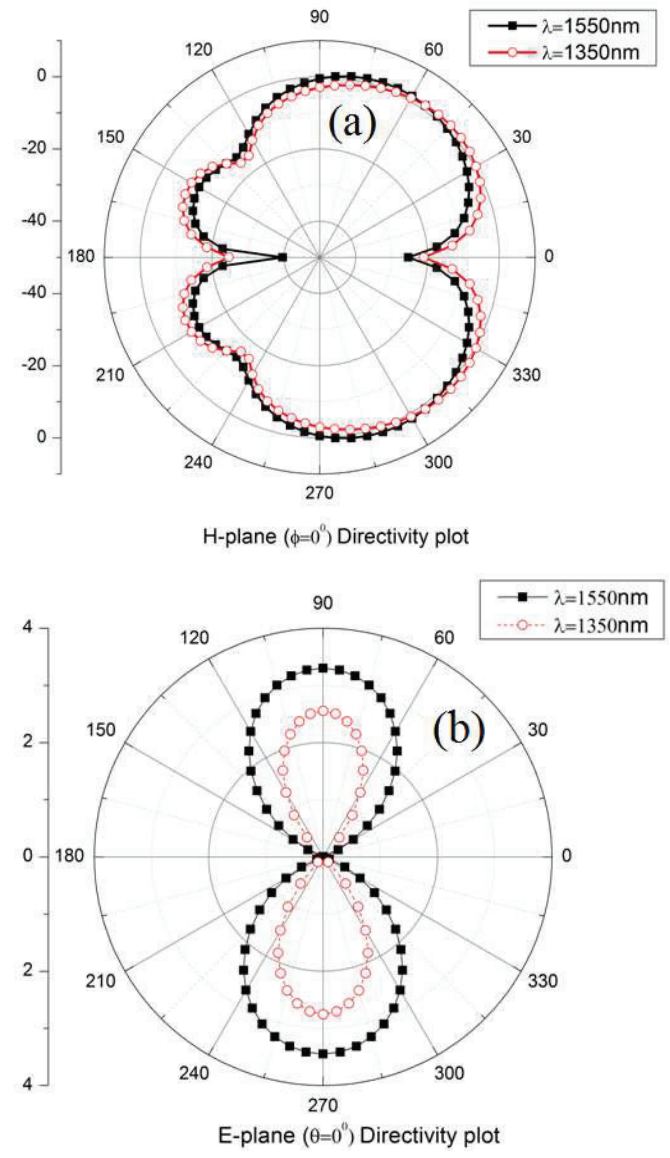

Figure 2: (a) Simulated E-Plane directivity plot (b) Simulated H-plane directivity plot

\section{References}

1. Amir Hosseini, Hamid Nejati, and Yehia Massoud, IEEE Int. Sym. On Cir. and Sys. (2008) 2346.

2. Volker J. Sorger, Rupert F. Oulton, Ren-Min Ma and Xiang Zhang, MRS Bulletin 37 (2012) 728. 